\title{
Fake Science - auch ein Problem in der Physiotherapieforschung?
}

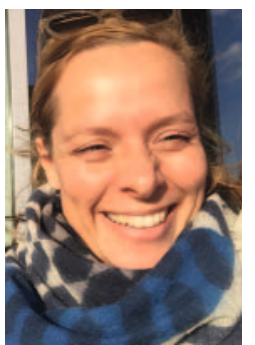

Dr. rer. nat. Kirstin-Friederike Heise, Herausgeberin physioscience
Korrespondenzadresse

Dr. rer. nat. Kirstin-Friederike Heise

Dept. of Movement Sciences - Motor Control and Neural

Plasticity Research Group, 3001 Leuven, Belgium

kirstin.heise@kuleuven.be

Bibliografie

DOI https://doi.org/10.1055/a-0749-0408

physioscience 2018; 14: 149-150

(c) Georg Thieme Verlag KG, Stuttgart · New York

ISSN 1860-3092
Täglich flattern mehr oder weniger dringliche Einladungen in meinen elektronischen Briefkasten, ein Manuskript in ein neues Journal einzureichen. Darunter wohlklingende Namen wie Annals of Physical Therapy, Annals of Biomedical Technology \& Engineering, Annals of Musculoskeletal Medicine, Journal of Life Science Innovations, International Journal of Sports and Exercise Medicine, etc. - die Liste ist nahezu endlos. Zumeist ignoriere ich diese E-Mails und lösche sie unmittelbar. Seit jedoch in diesem Sommer der unsägliche Begriff „Fake Science“, also der Betrug mit scheinwissenschaftlichen Arbeiten, das Thema in den Medien war, frage ich mich: Ist das auch ein Problem, welches die physiotherapeutische Forschung betrifft?

Hier gilt es zwei wesentliche Vorgänge des Betruges in der Wissenschaft zu unterscheiden: Auf der einen Seite das Betrügen mit manipulierten Daten und Ergebnissen, und auf der anderen Seite der Betrug mit vermeintlich wissenschaftlichen Publikationsplattformen. Während Ersteres zwar auch eine Folge der Spielregeln des Wissenschaftssystems sein kann, basiert es doch eher auf dem Handeln des Individuums und verstößt eindeutig gegen die Regeln der guten wissenschaftlichen Praxis [1]. Letzteres ist jedoch ausschließlich ein systemisches Problem, bewegt sich in einer legalen Grauzone und betrifft damit alle Akteure und Profiteure des Wissenschaftssystems - darüber schreibe ich in diesem Editorial.

Ohne wissenschaftliche Veröffentlichungen existiert man in der wissenschaftlichen Gemeinschaft quasi nicht. Hinzu kommt, dass einen Organisationen für Fördermittel ebenfalls zu einem großen Anteil anhand der Publikationsleistung messen. Ohne Publikationen kein Geld für Forschung, ohne Geld für Forschung keine Forschung, keine Publikationen, keine Teilnahme an internationalen wissenschaftlichen Konferenzen, etc.

So sind die Spielregeln und das gilt ebenfalls im Bereich der Physiotherapiewissenschaften. Ich würde sogar die These aufstellen wollen, dass es sich in unserem Bereich noch ein Stückchen gravierender darstellt, denn wir - ich spreche vor allem, aber nicht ausschließlich für den deutschsprachigen Raum - sind gerade erst dabei, unseren Fuß in die Tür der etablierten Fördermittelgeber zu setzen und die Relevanz unserer wissenschaftlichen Arbeit zu unterstreichen. Außerdem können wir unsere wissenschaftliche Gemeinschaft noch nicht so einwandfrei identifizieren, da die Physiotherapiewissenschaft noch kein etabliertes Feld ist, welches außerdem ein enormes Wachstum und eine Weiterentwicklung sowohl auf der Seite der Akteure als auch der Organe und Medien zur Veröffentlichung verzeichnet.

„Fake“ (gefälscht/falsch) oder „predatory“ (räuberisch), wie diese vermeintlichen Fachzeitschriften im Englischen genannt werden, drängen mit wissenschaftlichen Informationen auf den Markt und treffen den Nerv des Publikationsdrucks im Wissenschaftssystem. Das hat zur Folge, dass einerseits qualitativ minderwertige Arbeiten die Hürde zur Veröffentlichung nehmen und andererseits Wissenschaftler auf der Suche nach geeigneten Journals für ihre Arbeit in die Irre geleitet werden. Die E-Mail-Einladungen dieser Zeitschriften locken mit dem Versprechen einer garantierten Publikation, schnellem Peer-review-Verfahren innerhalb von wenigen Tagen oder Angeboten wie vom Discounter an der Ecke (zwei Publikationen zum Preis von einer).

Aufgrund des hohen Drucks zu publizieren bietet ein vermeintlich leichterer Weg mit kurzen Bearbeitungszeiten eine willkommene Möglichkeit, die eigene Arbeit in einem Fachjournal unterzubringen und damit seinen wissenschaftlichen Lebenslauf aufzuwerten. Die Medienberichte in diesem Sommer führten uns vor Augen, dass die Zahl der Wissenschaftler, die bewusst oder unwissend auf diesem Weg veröffentlichten, auch erfahrene und nicht nur unerfahrene Kollegen einschließt. Und das Problem betrifft selbstverständlich nicht ausschließlich den deutschsprachigen Raum.

Bei der Recherche für dieses Editorial fiel mir der Beitrag eines indischen Kollegen in die Hände, der darin dasselbe Problem beklagt und eindringlich dazu aufruft, solche trügerischen Medien zu meiden [2]. Wie drängend das beschriebene Problem ist, zeigt z. B. auch die im Juli dieses Jahres verabschiedete Richtlinie der kanadischen Regierung, mit dem Ziel der Stärkung der wissenschaftlichen Integrität [3]. Damit verbunden ist die explizite Ermutigung, adressiert an kanadische, im Auftrag der Regierung forschende Wissenschaftler, öffentlich über ihre Arbeit zu sprechen. Darüber hinaus betont die Richtlinie die Förderung einer 
Kultur, die die wissenschaftliche Integrität in der Forschung sowie die Erhöhung der Zuverlässigkeit von und des Vertrauens in Wissenschaft und Forschung der Regierung unterstützt [3].

Aber woran erkenne ich ein vertrauenswürdiges Journal und wie schütze ich mich vor betrügerischen Plattformen?

Der amerikanische Bibliothekar Jeffrey Beall kritisierte schon früh die uneingeschränkte Forderung nach freier Verfügbarkeit von Publikationen, bei der die Autoren für die Publikation ihrer Arbeit horrende Summen bezahlen [4]. Seine Kritik richtet sich gegen ein intransparentes Publikationssystem, welches dazu führt, dass betrügerische Plattformen Kapital aus dem Publikationsdruck schlagen können. Beall initiierte eine Liste mit potenziell räuberischen Verlage, Zeitschriften und Konferenzanbietern [5]. Diese ständig aktualisierte Liste bietet eine Hilfestellung bei der Identifizierung von Pseudofachzeitschriften.

Ein essenzieller Aspekt des wissenschaftlichen Publikationsprozesses ist die sorgfältige Untersuchung des Manuskriptes durch andere Wissenschaftler anhand eines Peer-Review-Verfahrens. Sie lesen das Manuskript kritisch und empfehlen entweder, es zur Publikation anzunehmen, abzulehnen oder - in den meisten Fällen - es nochmals zu überarbeiten. Ein ohne ein solches oftmals verblindetes Begutachtungsverfahren veröffentlichter Artikel wird im Grunde als nicht valide und nicht wissenschaftlich bewertet. Diese Arbeit, die die Gutachter unentgeltlich und zusätzlich zu allen anderen professionellen Aufgaben erledigen, hat damit einen unschätzbaren Wert für die Qualitätsgarantie in der Wissenschaft. Es ist evident, dass eine solche fundierte Begutachtung sowohl Expertise als auch Zeit bedarf.

Netzwerke (digitale und real weltliche) ermöglichen den Informationsaustausch und die Kooperation mit Kollegen auf regionaler, nationaler und internationaler Ebene. Die Kommunikation innerhalb solcher Netzwerke ist oftmals direkter und daher auch zeitlich unmittelbarer, was eine großartige Bereicherung für den wissenschaftlichen Diskurs darstellt und eine gewinnbringende Ergänzung zu den Printmedien bietet. Innerhalb solcher Netzwerke lassen sich Erfahrungen austauschen und bieten auch einen Beitrag zur Qualitätssicherung für wissenschaftliche Arbeiten. In diesem Zusammenhang möchte ich auf den Beitrag von A. Schäfer, C. Braun, B. Elsner, C. Kopkow und K. Lüdtke in dieser Ausgabe verweisen (s. S. 194 ff.).

Mentoring-Programme für angehende Wissenschaftler verdeutlichten bereits in anderen Wissenschaftsbereichen, dass sie wesentlich die Weichenstellung beeinflussen, ob Wissenschaftler erfolgreich, produktiv und zufrieden ihrer Forschung nachgehen können. Diese Programme unterstützen Neulinge dabei, ihren Weg zu finden und bieten eine effektive Möglichkeit, Kontakte und Kooperationen zu knüpfen und sich einen Namen in der wissenschaftlichen Gemeinschaft zu machen. Dies sind die wesentlichen Voraussetzungen, um sich mit den Spielregeln des Systems vertraut zu machen und nicht auf trügerische Angebote hereinzufallen. Strukturen für Mentoring-Programme erfordern selbstverständlich ebenfalls ökonomische Ressourcen und die Bereitschaft zu persönlichem Engagement. Andere wissenschaftliche Arbeits- bereiche zeigen jedoch, dass die Kultivierung effektiven Mentorships innerhalb des Wissenschaftsbetriebes langfristig dem gesamten System zugutekommt [6].

Ein Paradigmenwechsel zeichnet sich im Bereich des Open Access ab, also der freien Verfügbarkeit von wissenschaftlichen Ergebnissen. Ein Paukenschlag war in diesem Zusammenhang die Meldung Anfang September dieses Jahres, dass eine Reihe nationaler Forschungsförderorganisationen der Europäischen Kommission und dem Europäischen Forschungsrat folgen und Open-Access-Publikationen verpflichtend für Empfänger ihrer Fördergelder vorschreiben [7]. Wie schon angedeutet, ist das System der freien Verfügbarkeit nicht unumstritten. Bisher tragen vor allem die Autoren die Kosten für die freie Verfügbarkeit wissenschaftlicher Veröffentlichungen. Das soll und wird sich unweigerlich mit dieser maßgeblichen Neugestaltung der Förderauflagen ändern. Die Kritik, dass Bezahlschranken den Zugang zu Wissen verhindern und damit das grundlegende Universalitätsprinzip der Wissenschaft untergraben, treibt diese fundamentalen strukturellen Umwälzungen an. Nur dann, wenn Ergebnisse diskutiert, auf Nachvollziehbarkeit hin überprüft, reproduziert und gegebenenfalls widerlegt werden können, sind sie als wissenschaftlich zu bezeichnen. Der Grundcharakter der Wissenschaft als Institution besteht in der kritischen Betrachtung und kann sich folglich nur dann entfalten, wenn diese Verzahnung aus kritischer Reflexion, Überprüfung und Basierung neuer Untersuchungen auf bestehendem Wissen uneingeschränkt möglich ist.

In diesem Sinne wünsche ich Ihnen viel Vergnügen beim kritischen Lesen der aktuellen Ausgabe der physioscience. Vielleicht nehmen Sie sich auch mal die Zeit, um die Liste der Gutachter und der Kollegen im wissenschaftlichen Beirat anzuschauen, die einen großen Anteil an der Güte dieser Fachzeitschrift haben.

Literatur

[1] Deutsche Forschungsgemeinschaft. Sicherung guter wissenschaftlicher Praxis - Denkschrift. 2013 www.dfg.de/download/pdf/dfg_im_profil/ reden_stellungnahmen/download/empfehlung_wiss_praxis_1310.pdf (22.09.2018)

[2] Samuel A. An Indian physiotherapist's suggestions to keep out of fake journals. Physiother - J Indian Assoc Physiother 2018; 12: 43. doi: 10.4103/PJIAP.PJIAP_32_17

[3] Government of Canada. Model policy on scientific integrity. 2018 www. ic.gc.ca/eic/site/052.nsf/eng/00010.html (22.09.2018)

[4] Beall J. Predatory publishers are corrupting open access. Nature 2012; 489: 179-179. doi:10.1038/489179a

[5] Beall J. Beall's List of Predatory Journals and Publishers. 2018 www.be allslist.weebly.com/\# (22.09.2018)

[6] Kuhn C, Castaño Z. Boosting the career development of postdocs with a peer-to-peer mentor circles program. Nat Biotechnol 2016; 34: 781 783. doi: org/10.1038/nbt.3631

[7] Schiltz M. Science without Publication Paywalls: cOAlition S for the Realisation of Full and Immediate Open Access. Front Neurosci 2018; 12 : 656. doi: $10.3389 /$ fnins.2018.00656 